\title{
DIAGNOSTIC FEATURES OF DYSFUNCTION IN CYTOKINE AND SYMPATHOADRENAL SYSTEMS WITH ISCHEMIC STROKE
}

10.36740/WLek202010124

\author{
Volodymyr S. Lychko \\ MEDICAL INSTITUTE OF SUMY STATE UNIVERSITY, SUMY, UKRAINE
}

\begin{abstract}
The aim: Was a comprehensive study of the changes in features like structural and functional parameters of brain tissue, cytokine profile and $\beta$-adrenoceptor in the acute period of ischemic stroke (IS) to optimise diagnosis and treatment. The study was conducted at the Department of Neurosurgery and Neurology at the Medical Institute of Sumy State University.

Materials and methods: Measurements of complex dielectric constant (CDC) were performed by EHF dielectrometry. Determination of plasma levels of interleukin-6 (IL-6) and tumour necrosis factor-a (TNF-a) was performed by solid-phase enzyme-linked immunosorbent assay. The work was based on the materials of a comprehensive examination of 350 patients with the first in the life of $I S$ on the 1st, 10th and 21 st day of the disease.

Results: Cerebral hypoxia leads to a significant increase in the CDC of erythrocyte membranes (about $15 \%$ ), which may lead to a rise in the volume fraction of water in cell suspensions due to damage of their membrane-receptor complex (MRC). The revealed connections between changes of CDC of patients with IS and decrease insensitivity of their MRC to adrenergic drugs testify in favour of damaging action of hypoxia. An imbalance in the cytokine system and their relationship to the sympathoadrenal system has been identified.

Conclusions: There is a strong association of mechanisms that damage the MRC of cells during acute cerebral ischemia with functional changes in both the sympathoadrenal and cytokine systems. Identified links in the pathogenesis of IS should be used for early diagnosis of the disease.
\end{abstract}

KEY WORDS: receptor, membrane, dysfunction, cerebral ischemia, adrenergic reactivity

Wiad Lek. 2020;73(10):2233-2237

\section{INTRODUCTION}

Hyper-reactivity of the centres in the sympathetic nervous system with the stimulation of numerous neurohumoral factors plays a leading role in the pathogenesis of ischemic stroke (IS) [1,2]. The most important are such biogenic monoamines as norepinephrine, adrenaline, dopamine, serotonin and others. It is known that their level is regulated by the influence of monoamine oxidase (MAO) [3, 4]. Increased $\mathrm{MAO}$ activity during IS is associated with increased levels of catecholamines (CA) [5].

In the regulation of the activity of the sympathoadrenal system (SAS) a significant place is given to adrenoceptors (ARs). The number of ones always compensatory increases during stress, atherosclerosis, hypertension, endothelial dysfunction $[1,6]$.

Hypersympathicotonia is an influential factor that links the physiological regulation of vascular tone, cardiac activity and stable pressor response. This pool elevation in plasma CA is an independent marker of a high risk of acute cardiovascular and cerebrovascular events $[1,2,4]$. At the same time, the value of tissue SAS in the mechanisms of IS development has not been fully established to date.

Structural elements of blood are an integral part of tissue SAS, taking a direct part not only in ensuring adequate microcirculation but also in modulating the growth and differentiation of tissues, physiological fluctuations of hemodynamic and neuroendocrine constants [7]. Erythrocytes have active immunoregulatory properties and are considered as an adequate model for studying various diseases of polygenic nature, which include IS. They have an active membrane-receptor complex (MRC), more represented by $\beta_{2}$-ARs $[7,8]$.

The effect of adrenergic drugs (AD) on cells is inextricably linked with the concept of adrenergic reactivity (AR), which characterizes the systemic response of the body in response to changes in the activity of SAS $[9,10]$.

Summarizing the above, it can be argued that during IS, the relationship between plasma CA concentrations in plasma, an affinity for ARs, their expression on the surface of erythrocytes and intracellular accumulation of CA [11]. As the membrane-receptor dysfunction progresses, there is a violation, and then a complete separation of the mechanisms of production, transport and deposition of hormones, which leads to a complete failure of the regulatory axis «hormone-receptor». This is quite probable because the relationship between the expression of ARs, their affinity and the concentration of CA over the receptor field, developed by the mechanism of up- / down-regulation and uncoupling which are typical pathological mechanisms [12].

One of the primary triggers of the cytokine system in the acute period of IS is interleukin-6 (IL-6), which always accompanies the local inflammatory reaction. It has a diverse 
and potent effect on most organs and systems, primarily immune and endocrine [13].

IL- 6 acts as a powerful activator of the hypothalamic-pituitary-adrenal system. It is crucial that its secretion is enhanced under the influence of stress on the background of activation of the SAS, and is regulated by the CA on the principle of positive feedback. Elevated levels of IL-6 in the blood are observed during severe inflammatory processes, infections, injuries, i.e. conditions when the secretion of an antidiuretic hormone may be impaired [14].

SAS is directly related to the immune system through the innervation of many immune organs (such as the spleen and thymus). Stress or its simulation by the introduction of exogenous adrenaline increases the level of endogenous IL-6 in experimental animals. However, the previous introduction by $\beta$-adrenal blockers $(\beta-\mathrm{AB})$ prevents the development of this reaction. It is assumed that the stimulation of IL- 6 secretion is also mediated by $\beta$-ARs.

In a study [15] it was shown that the introduction of adrenaline increases the level of IL- 6 in the blood. The maximum concentration of CA in the blood directly correlated with the level of IL-6. These data confirm that stress provokes hypersecretion of IL- 6 due to stimulation of $\beta$-ARs.

Expression of tumour necrosis factor- $\alpha$ (TNF- $\alpha$ ) over time coincides with the activation of IL- 6 and precedes the development of a local inflammatory reaction in the area of ischemia. High levels of these pro-inflammatory cytokines are found not only in the macrophages of the infarct zone but also in the neurones of the necrosis nucleus, as well as in the penumbra. In addition, TNF- $a$ causes the expression of pro-adhesive endothelial molecules, which leads to the concentration of leukocytes, their adhesion and migration from capillaries to the brain, the development of local inflammation and increase in the size of the lesion [16].

Today, we have many unresolved issues regarding the diagnosis and understanding of the leading mechanisms of IS. These include uncertainty with the state of MRC cells, which become the first target for numerous pathological cascades under the condition of acute cerebral ischemia and as a consequence there are functional changes in the SAS and cytokine system of the body. The significance of tissue SAS in the mechanisms of IS development has not been fully established till date. At present, there is still no single approach to the method of determining the activity of SAS; therefore, there is no unambiguous answer about the correct choice of its diagnostic tools.

\section{THE AIM}

The aim was a comprehensive study of the features of changes in the structural and functional parameters of brain tissue, cytokine profile and $\beta$-ARs in the acute period of IS to optimise diagnosis and treatment.

\section{MATERIALS AND METHODS}

Measurements of complex dielectric constant (CDC, $\Delta \varepsilon^{c}$ ) were performed by EHF dielectrometry. Determination of plasma levels of IL- 6 and TNF- $\alpha$ was performed by solid-phase enzyme-linked immunosorbent assay.

The work was based on the materials of a comprehensive examination of 350 patients with the first in the life of IS on the 1st, 10th and 21st day of the disease. The criteria for selecting patients were: age from 46 to 79 years; the first in the life of IS with localisation in the pool of blood supply to the middle cerebral artery; hospitalisation of the patient up to 24 hours from the beginning of the disease; MRI results that ruled out intracranial haemorrhage or other non-ischemic brain damage.

Males accounted for 185 patients (52.8\%), females - 165 (47.2\%). The mean age of the examined patients was $65.2 \pm$ 0.7 years (range from 46 to 79 years). As a comparison group, 30 conditionally healthy individuals (17 men and 13 women, mean age $-60.4 \pm 0.8$ years), who are homogeneous in sex and age with a group of patients with IS, were additionally examined.

All human studies were performed according to the Declaration of Helsinki (1964, last revision of the 64th WMA General Assembly, Brazil, 2013).

The severity of the condition and the degree of the neurological deficit was determined using the stroke scale of the US National Institutes of Health (NIHSS) with a score in the first hours of the disease, in the dynamics of treatment on the 10th and 21st day.

Based on this, all patients were divided into 2 clinical groups: 1 st $(\mathrm{n}=183)$ - patients in a state of moderate severity (mean score on the NIHSS scale $11.74 \pm 0.33$ ); $2 \mathrm{nd}(\mathrm{n}=167)$ - patients with severe IS (mean score on the NIHSS scale $24.06 \pm 0.29$ ).

\section{RESULTS AND DISCUSSION}

Given the pathogenetic significance of functional disorders of the adrenergic receptor system in the acute period of IS using EHF dielectrometry, the dynamics of changes in $\beta$-AR and the values of CDC under the action of biologically active substances (BAS) was studied.

As a result, it was found that the relative values of $\Delta \varepsilon^{\prime}$ were differentiated by groups (Table 1).

In the control group, a significant decrease in the dielectric constant $\Delta \varepsilon^{\prime}$ was observed after exposure to adrenaline solution. In patients with IS, the response to adrenaline was virtually absent, which may be due to a decrease in the sensitivity of $\beta$-ARs erythrocytes to stimulants due to their desensitisation. However, according to the magnitude of changes in $\Delta \varepsilon^{\prime}$ in the group of patients with moderate severity, it can be stated that their erythrocytes were more reactive than in the group of patients with a more severe variant of IS.

A similar effect was also observed when comparing the dynamics of changes in the values of $\Delta \varepsilon^{\prime}$ erythrocyte suspensions under the influence of $\beta$ - $A B$ propranolol depending on the severity of IS.

Effects in the control group in $100 \%$ of cases had a negative sign, the magnitude of the impact in $44 \%$ of cases exceeded $10 \%$ relative to samples with an equal volume addition of $0.9 \%$ saline. In the group of patients with severe IS, all changes were within error. In the group of patients 
Table 1. Dynamics of values of $\Delta \varepsilon^{\prime}$ erythrocyte suspensions after exposure to adrenaline in patients with IS depending from the severity of the disease

\begin{tabular}{ccc}
\hline Group of patients & The term of the study & The value of $\boldsymbol{\Delta \boldsymbol { \varepsilon } ^ { \prime }}$ \\
\hline 1 & 2 & 3 \\
\hline \multirow{3}{*}{1 Group } & 1 st day & $0,21 \pm 0,08$ \\
\cline { 2 - 3 } & 10 th day & $0,33 \pm 0,04$ \\
\hline \multirow{2}{*}{2 Group } & 21 st day & $0,35 \pm 0,1$ \\
\hline & 1 st day & $-0,48 \pm 0,18$ \\
\hline & 10 th day & $-0,33 \pm 0,09$ \\
\hline
\end{tabular}

Control group

$-1,21 \pm 0,15$

Note. All indicators are reliable for control $(p<0,05)$.

Table 2. General levels of pro- and anti-inflammatory cytokines in the serum of patients with $\mathrm{IS}$ in the dynamics of treatment $(\mathrm{M} \pm \mathrm{m}, \mathrm{pg} / \mathrm{ml})$

\begin{tabular}{ccccccc}
\hline \multirow{2}{*}{ Group of patients } & \multicolumn{3}{c}{ II-6 } & \multicolumn{3}{c}{ TNF-a } \\
\cline { 2 - 6 } & $\mathbf{1}$ st day & $\mathbf{1 0}$ th day & $\mathbf{2 1}$ st day & $\mathbf{1}$ st day & $\mathbf{1 0}$ th day & $\mathbf{2 1}$ st day \\
\hline All & $32,75 \pm 3,44$ & $16,13 \pm 0,77$ & $13,2 \pm 0,66$ & $16,13 \pm 0,77$ & $13,2 \pm 0,66$ & $6,97 \pm 0,44$ \\
\hline 1 st Group & $34,58 \pm 2,77$ & $15,89 \pm 1,05$ & $14,57 \pm 0,38$ & $15,89 \pm 1,05$ & $14,57 \pm 0,38$ & $8,62 \pm 0,24$ \\
\hline 2 nd Group & $33,78 \pm 3,03$ & $16,38 \pm 0,78$ & $11,84 \pm 0,67^{*} \wedge$ & $16,38 \pm 0,78$ & $11,84 \pm 0,67^{*} \wedge$ & $5,33 \pm 0,37$ \\
\hline Control Group & & $3,51 \pm 0,28$ & & & $4,12 \pm 0,34$ \\
\hline
\end{tabular}

Notes:

All indicators are reliable for control $(p<0,05)$;

* $-p<0.05$ between the 1 st and 2 nd groups of patients;

$\wedge-p<0.05$ before and after treatment within the group.

with moderate disease, a reaction to $\beta-\mathrm{AB}$ was observed; however, the sign of the effect was opposite to the control indicator, as in the case of adrenaline.

As for the combined action of adrenaline and propranolol, when the adrenaline was added after previous incubation of cells with $\beta-A B$, the reactions were observed only in $46.3 \%$ of cases in the group of patients with moderate IS. No responses in the control group were expected due to previous $\beta$-ARs blockade.

In all samples of patients with moderate severity, there was a tendency to increase the absolute values of CDC on the background of prescribed treatment, which indicates changes in the levels of hydration of erythrocytes. All this indicates an increase in the amount of free water in the samples and a decrease in the amount of bound. This assumption can be made based on the fact that the dynamics of the disease reduces the thickness of the pre-membrane layer to $20 \%$.

This affects the rate of metabolic processes in the system «cell-intercellular environment», thereby changing the mode of functioning of both the cell and its MRC.

Correlation analysis of the relationship between CDC values and the total clinical score on the NIHSS scale also showed some features. There was a significant negative correlation between the values of $\Delta \varepsilon$ and the level of the neurological deficit on the NIHSS scale in patients with IS at the onset of the disease $(\mathrm{r}=-0.83 ; \mathrm{p}<0.05)$. In the dynamics of observation between these indicators also remained a significant correlation, which did not lose its strength and direction $(r=-0.77 ; p<0.05)$.
Given that IS is a classic example of an aseptic inflammatory reaction that develops after the formation of a zone of necrosis, a study of cytokine status in the dynamics of drug treatment.

When analyzing the average values of IL- 6 in patients with IS, it was found that the highest levels of IL- 6 in the serum were determined in patients with the severe process during hospitalization (Table 2). At the same time, their levels exceeded the indicators of the control group by almost ten times $(34.33 \pm 2.9 \mathrm{pg} / \mathrm{ml}$ in severe IS against $3.51 \pm$ $0.28 \mathrm{pg} / \mathrm{ml}$ in the control group, $\mathrm{p}<0.001)$. In patients with moderate IS, IL-6 levels exceeded the control group 5 times $(17.62 \pm 2.3 \mathrm{pg} / \mathrm{ml}, \mathrm{p}<0.001)$. All detected changes indicate the maximum intensity of the stress-implementing immune system in severe IS.

In all subgroups, the severity of both groups showed a significant decrease in IL-6 levels after treatment relative to the indicators on the 1 st day during hospitalization $(p<0.05)$. In patients with moderate IS, IL-6 levels on the 10th day of the disease were close to each other in the 1 st and 2 nd groups. Their significant decrease compared to the indicators on the 1st day after the onset of acute neurological symptoms occurred by $15.5 \%$ and $37.3 \%$, respectively. This can be explained by the fact that with a small lesion of brain tissue, the stress-realization reaction can limit the physiological stress-limiting systems, and it is not yet distressful.

In all groups of patients with IS of severity revealed elevated levels of TNF- $\alpha$ relative to control $(p<0,05)$. The maximum 
values of the indicator were observed in severe patients in the 1st and 2 nd groups $(20.15 \pm 0.71$ and $21.06 \pm 0.50 \mathrm{pg} / \mathrm{ml}$, respectively), which indicates the maximum intensity of the neuroendocrine response in IS.

The dynamics showed a tendency to decrease the levels of TNF- $\alpha$ on the 10th day of IS relative to baseline values recorded on the 1st day, but it did not reach the degree of reliability. Evaluating the dynamics of TNF- $\alpha$ levels on the 21st day of treatment, the closest values to the control were observed in the group of patients with the moderate condition $(\mathrm{p}<0.05)$.

Correlation analysis of the relationship between the activity of the sympathoadrenal and cytokine systems, the severity of patients with IS revealed many features.

During the statistical processing of the relationships between the levels of IL- 6 and TNF- $\alpha$ in patients with IS, a significant direct correlation was found between these indicators on the 1 st day of the disease $(r=+0.91 ; p<0.05)$, which confirms the unidirectionality of their action in the induction of pathological processes during IS.

Inverse correlations of values of IL- 6 and TNF- $\alpha$ in blood serum and CDC $(r=-0.78 ; \mathrm{p}<0.05$ and $\mathrm{r}=-0.85 ; \mathrm{p}<0.05$, respectively) were also revealed, which indicates the inhibitory effect of pathophysiological processes associated with the activation of the cytokine system on the functional state of the adrenoceptors system of patients with IS.

The dependence of the levels of factors of the cytokine system on the severity of the disease is evidenced by the identified correlations. Thus, in the debut of IS were recorded direct links between the levels of IL-6, TNF- $\alpha$ in serum and the total clinical score $(\mathrm{r}=+0.895 ; \mathrm{p}<0.05$ and $\mathrm{r}=+0.9 ; \mathrm{p}<0,05$ respectively).

The reduced ability of ARs on erythrocyte membranes to bind blockers due to the desensitization effect due to MRC damage is evidenced by the established direct, reliable relationship between the area of the infarct zone and the values of $\beta-A R(r=$ $+0.88 ; \mathrm{p}<0.05)$.

\section{CONCLUSIONS}

Summarising the above, it is clear that there is a strong link between mechanisms that damage the MRC of cells during acute cerebral ischemia with functional changes in both the sympathoadrenal and cytokine systems. At the same time, under conditions of hypo-perfusion, acute endothelial dysfunction, energy deficiency, free radical and mediator aggression develop very quickly.

Before an acute vascular catastrophe, which is IS, changes in the adrenoceptor and cytokine systems adversely affect the course of the disease, with morphological cell damage rapidly joining. They very quickly change the synthetic ability to catabolic, increasing the permeability of their membranes to pro-inflammatory agents with a violation of their barrier functions.

\section{REFERENCES}

1. Akil E., Tamam Y., Akil M.A. et al. Identifying autonomic nervous system dysfunction in acute cerebrovascular attack by assessments of heart rate variability and catecholamine levels. Journal of Neurosciences in Rural Practice. 2015; 6 (2): 145-150.
2. De Raedt S., De Vos A., De Keyser J. Autonomic dysfunction in acute ischemic stroke: An underexplored therapeutic area? Journal of the Neurological Sciences. 2015; 348 (1-2): 24-34.

3. Choi-Kwon S., Ko M., Jun S.E. et al. Post-stroke fatigue may be associated with the promoter region of a monoamine oxidase a gene polymorphism. Cerebrovascular Diseases. 2017; 43 (1-2): 54-58.

4. Hama S., Murakami T., Yamashita H. et al. Neuroanatomic pathways associated with monoaminergic dysregulation after stroke. International Journal of Geriatric Psychiatry. 2017; 32 (6): 633-642.

5. Miro-Mur F., Laredo C., Renu A. et al. Neuroanatomic pathwaysand circulating leukocyte subtypes in stroke patients treated with reperfusion therapy. Brain Behav Immun. 2018; 70: 346-353.

6. Huck J.H.J., Freyer D., Bottcher C. et al. De novo expression of dopamine D2 receptors on microglia after stroke. Journal of Cerebral Blood Flow and Metabolism. 2015; 35 (11): 1804-1811.

7. Hamner J.W., Tan C.0., Lee K. et al. Sympathetic control of the cerebral vasculature in humans. Stroke. 2010; 41 (1): 102-109.

8. Hu C.M.J., Zhang L., Aryal S. et al. Erythrocyte membrane-camouflaged polymeric nanoparticles as a biomimetic delivery platform. Proceedings of the National Academy of Sciences of the United States of America. 2011; 108 (27): 10980-10985.

9. Kobilka B.K. Structural insights into adrenergic receptor function and pharmacology. Trends in Pharmacological Sciences. 2011;32 (4):213-218.

10. Laureys G., Clinckers R., Gerlo S. et al. Astrocytic beta(2)-adrenergic receptors: From physiology to pathology. Progress in Neurobiology. 2010; 91 (3): 189-199.

11. Kim G.S., Yang L., Zhang G.Q. et al. Critical role of sphingosine-1phosphate receptor- 2 in the disruption of cerebrovascular integrity in experimental stroke. Nature Communications. 2015; 6: 1-15.

12. Webster J.C., Cidlowski J.A. Down-regulation of the glucocorticoid receptor - a mechanism for physiological adaptation to hormones. Brain Corticosteroid Receptors: Studies on the Mechanism, Function, and Neurotoxicity of Corticosteroid Action. 1994; 746: 216-220.

13. Erta M., Quintana A., Hidalgo J. Interleukin-6, a major cytokine in the central nervous system. International Journal of Biological Sciences. 2012; 8 (9): 1254-1266.

14. Winter C.D., Pringle A.K., Clough G.F., Church M.K. Raised parenchymal interleukin-6 levels correlate with improved outcome after traumatic brain injury. Brain. 2004; 127: 315-320.

15. Gornikiewicz A., Sautner T., Brostjan C. et al. Catecholamines up-regulate lipopolysaccharide-induced IL-6 production in human microvascular endothelial cells. Faseb Journal. 2000; 14 (9): 1093-1100.

16. Chen A.Q., Fang Z., Chen X.L. et al. Microglia-derived TNF-alpha mediates endothelial necroptosis aggravating blood brain-barrier disruption after ischemic stroke. Cell Death \& Disease. 2019; 10: 1-18.

The research is a part of the comprehensive research program of Medical institute of Sumy State University, The Ministry of Education of Ukraine, Department of Neurosurgery and Neurology "Сучасні аспекти клініко-лікворологічної діагностики в неврологіï, нейрохірургіï, психіатрії» (State registration number 0110U007827; 2018-2021).

ORCID and contributionship:

Volodymyr S. Lychko: 0000-0001-5518-5274 ${ }^{A, B, C, D, E, F}$

\section{Conflict of interest:}

The Author declares no conflict of interest. 


\section{CORRESPONDING AUTHOR}

Volodymyr S. Lychko

Medical Institute of Sumy State University

1 Sanatorna st., 40018 Sumy, Ukraine

tel: +380662550120

e-mail: volodlychko@gmail.com

Received: 21.05 .2020

Accepted: 31.08 .2020

A - Work concept and design, B - Data collection and analysis, C - Responsibility for statistical analysis,

D-Writing the article, $\mathbf{E}$-Critical review, $\mathbf{F}$ - Final approval of the article 